\title{
Post Operative Acute Urinary Retention Following Lateral Internal Sphincterotomy
}

Elroy Patrick Weledji* and Divine Eyongeta ${ }^{1}$

${ }^{1}$ Faculty of Health Sciences, University of Buea, Cameroon

\begin{abstract}
Autonomic dysfunction is a common complication in colorectal surgery. Approximately a third of patients undergoing anorectal surgery develop acute urinary retention. We report a case of acute urinary obstruction post lateral internal sphincterotomy for chronic anal fissure in a 61-year old man with no antecedent history of bladder outflow obstruction nor benign prostatic hyperplasia on examination. We suggest the importance of preoperative catheterization of high risk patients undergoing anorectal surgery and avoiding the insertion of haemostatic anal pack.
\end{abstract}

Keywords:Lateral internal; Sphincterotomy; Acute urinary retention

\section{Introduction}

Acute urinary retention is a common post operative complication following surgery on benign anorectal diseases and increases hospital stay [1]. The incidence of post operative urinary retention after anorectal surgery is $15 \%$ (range $1-52 \%$ ) [2]. It is most common after haemorrhoidectomy but a 30\% incidence after lateral internal sphincterotomy [3].Gender-specific pathologies such as benign prostatic hyperplasia among others give a higher incidence of postoperative urinary retention in males $(4.7 \%)$ than in women $(2.9 \%)$. The risk increasing by 2.5 times in patients over $50 \mathrm{yrs}$ of age due to age - related progressive neuronal degeneration leading to bladder dysfunction [3]. It may be clinically apparent which of these causes is the most likely, but urodynamic investigation will demonstrate the difference between obstruction and atonia. If the cause is prostatic hyperplasia, then a transurethral prostatatectomy can be performed but if the cause is atonia, then a period of some weeks of catheterization will often allow the bladder to recover tone and permit adequate voiding [4].

\section{Case Presentation}

A 61 -year -old school teacher was admitted for an elective lateral internal sphincterotomy for a 1yr history of a chronic anal fissure. This presented as a progressive anal pain on defecation and associated blood staining of the toilet paper. His bowel habit was regular, twice per day but complained of a history of straining at stool. He was otherwise well with no anorexia nor weight loss. He has a past medical history of peptic ulcer disease. General and abdominal examination was unremarkable. Rectal examination revealed a chronic anal fissure at 6 'oclock associated with a skin tag (sentinel pile) but pain and anal spasm prevented a digital examination. A basic full blood count was normal. Examination under anesthesia using ketamine revealed a posterior chronic anal fissure with no evidence of an underlying abscess. The prostate was not palpably enlarged or otherwise. Proctosopy revealed no rectal mucosal lesion. An open lateral internal sphincterotomy to the length of the fissure was performed and haemostatic vaseline- rolled gauze was inserted into the anal canal. The procedure lasted 20 mins and the patient received $\sim 500$ $\mathrm{mls}$ of normal saline preoperatively. The post operative blood pressure was $135 / 65 \mathrm{mmHg}$, pulse $98 / \mathrm{min}$, respiratory rate $25 / \mathrm{min}$ and the temperature was $36.5^{\circ} \mathrm{C}$. The anal pack was removed after about $12 \mathrm{hrs}$. The following day he had no further pain following defecation and required just paracetamol for mild wound pain. He however gradually complained of difficulty in passing urine and voided but in small amounts. This was associated with some lower abdominal discomfort. He was advised to ambulate and continued on a central analgesic (paracetamol 1gm t.d.s), an antibiotic metronidazole $500 \mathrm{mg}$ t.d.s and maintained on the laxative, lactulose 1 sachet b.d. By the end of the day he complained of severe lower abdominal pain. Physical examination revealed a pelvic mass extending to the umbilicus. It was dull and tender on percussion and the lower border was not palpable consistent with a distended bladder. The diagnosis of an acute urinary retention was made. Under sterile technique and antibiotic (ciprofloxacin 500 mg bd) cover he was urethrally catheterized with a size -14 simplastic Foley catheter and his acute urinary retention relieved. A trial removal of catheter after $24 \mathrm{hrs}$ failed as he returned into retention about $12 \mathrm{hrs}$ later. A urethral catheter was reinserted and he was discharged home to return in 1 week for a further trial removal. However, a prostate specific antigen (PSA) test returned with a high level of $19 \mathrm{ng} / \mathrm{ml}$ (normal $<4.0 \mathrm{ng} / \mathrm{ml}$ ). An ultrasound scan of the prostate and renal tract was requested and the patient referred to the urologist but abdicated.

\section{Discussion}

Lateral internal sphincterotomy is a suitable and effective treatment for chronic anal fissure in patients who do not respond to conservative treatment. It is associated with significantly less post operative discomfort and increased healing rate with less morbidity [5]. However, acute urinary retention is common for various reasons [1-3]. The cause of this patient's post operative urinary retention remains elusive. The patient had no antecedent history of bladder outflow obstruction not irritative symptoms and physical examination did not reveal an obstructing benign prostatic hyperplasia not the abnormal hard, nodular cancerous prostate. Urethral catheterization and prostatitis may also give a high PSA level.

*Corresponding author: Elroy Patrick Weledji, FRCS, Senior Lecturer in Anatomy and Clinical Surgery, Faculty of Health Sciences, University of Buea, Cameroon; Tel: +237- 333221 34; E-mail:: elroypat@yahoo.co.uk

Received January 21, 2015; Accepted February 27, 2015; Published March 10 2014

Citation: Weledji EP, Eyongeta D (2014) Post Operative Acute Urinary Retention Following Lateral Internal Sphincterotomy; Outcome of Surgical Excision with Intralesional corticosteroid and Identification of Risk Factors for Recurrence. Surgery Curr Res 5: 221. doi:10.4172/2161-1076.1000221

Copyright: @ 2014 Weledji EP, et al. This is an open-access article distributed under the terms of the Creative Commons Attribution License, which permits unrestricted use, distribution, and reproduction in any medium, provided the original author and source are credited. 
It is known that the amount of intravenous fluids may influence the development of post operative urinary retention [3]. In patients undergoing hernia surgery and anorectal surgery intravenous administration of more than $750 \mathrm{mls}$ of fluids during the perioperative period increased the risk by 2.3 times compared to other surgeries [6]. Excessive infusion of intravenous fluids can lead to over distension of the bladder, especially in patients under spinal anesthesia whose bladder filling perception is abolished. It is also known that over distension inhibits detrusor function and the normal micturition reflex would not be restored even after emptying the urinary bladder with a catheter[7] The fact that bladder volume greater than $270 \mathrm{ml}$ represents a risk factor for post operative retention may explain may explain why a prolonged duration of surgery, is also a risk factor: 2.3 The patient received $500 \mathrm{mls}$ of fluid perioperatively and the surgical procedure was short.

General anaesthetic agents cause bladder atony by interfering with the autonomic nervous system [3]. In this patient, ketamine was used which has less effect on the autonomic nervous system than the standard anaesthetic agents [8]. The patient did not receive any systemic opioids that may inhibit the release of acetycholine from the parasympathetic sacral neurons that control detrusor contractility and also increase the incidence of acute urinary retention [7]. However, as post operative pain, rectal distension and anal dilatation increase sympathetic tone, the haemostatic anal pack may have exacerbated all these. It is advisable to avoid inserting an anal haemostatic pack but if necessary, an indwelling urinary catheter should be considered for at least 24 hours to avoid acute urinary retention [9].

Anxiety is thought of as a risk factor but the use of an anxiolytic agent is not effective in the treatment of postoperative urinary retention [3]. However, the para-sympathomimetic agent, bethanechol, in a dose of 10 mg subcutaneously, significantly lowers the incidence of postoperative urinary catheterization and should be considered as initial treatment of postoperative urinary retention following anorectal surgery [10]. It seldom works on the detrusor that is exhausted by obstruction or rendered unstable from whatever causes [4]. Further studies are needed to establish the role of $\alpha$-antagonists in the prevention of post operative urinary retention among patients undergoing anorectal surgery [11].

Post operative urinary retention in ambulatory surgery (low risk patients) is usually treated with in-out catheterization, but for anorectal surgery most authors suggest 5 days with a range between 3-10 days $[12,13]$. The incidence of urinary tract infection after anorectal surgery and 5 days of catheterization ranges between $42 \%$ and $60 \% .14$ For major complicated surgery and with extensive perineal and rectal dissection, bladder catheterization is required for a longer period of time according to clinical conditions [13].

The patient's high PSA, although not of $100 \%$ specificity may also suggest an underlying prostate cancer. However, It may also simply be a transient rise due to the acute urinary retention and catheterization $[14,15]$. Prostatitis and benign prostatic adenoma may also give high readings. Therefore a repeat PSA will be required after this crisis and if persistently positive or rising a transrectal ultra sound-guided prostatic biopsy of any suspicious lesion would be indicated [15]. It is possible but uncommon for prostate cancer to have their first presenting feature in this manner without a history of pre-retention or irritation.

\section{Conclusions}

It is vital to discuss preoperatively the potential bladder complications with all males undergoing pelvic and perineal surgery.
Since post operative urinary retention after anorectal surgery is common, it makes sense to routinely catheterize the high risk patients preoperatively. This would prevent post operative urinary retention until normal bladder function returns. Post operative haemostatic anal packs should be avoided but if required an indwelling catheter should be considered as well.

\section{References}

1. Toyonaga T, Matsushima M, Sogawa N, et al. (2006) Postoperative urinary retention aftersurgery for benign anorectal disease: Potential risk factors and strategy for prevention. Int J Colorectal Dis 21:676-82.

2. Zaheer S, Reilly WT, Pemberton JH, Ilstrup D (1998) Urinary retention after operations for benign anorectal diseases. Dis Colon Rectum 41: 696_704.

3. Baldini G, Bagry H, Aprikian A, Carli F (2009) Post operative urinary retention, Anaesthesiology 110: 1139-1157.

4. Blandy $J$ (2009) The Bladder: Disorders of Micturition. (6 $6^{\text {th }}$ edn.) Wiley Blackwell publishers, UK.

5. Schofield PF (1997) latrogenic disease. Surgery of the Colon and Rectum.

6. Bailey HR, Ferguson JA (1976) Prevention of urinary retention by fluid restriction following anorectal operations. Dis Colon Rectum 19: 696_704.

7. Yoshimura N, Chancellor MB (2002) Physiology and pharmacology of the bladder and urethra, Campbell-Walsh Urology. (9th edn.) Edited by Wein AJ Kavoussi LR, Novick AC, Partin AW, Peters CA (2006) Elsevier Philadelphia: 1922_1979.

8. Varrassi G, Panella L, Piroli A, Marinangeli F, Varrassi S et al. (1994 The effects of perioperative ketorolac infusion on postoperative pain and endocrine-metabolic response. Anesth Analg 78: 514-519.

9. Agarwal AK, Shenfine J, El- Khalafi, Leaper DJ (2006) Colorectal surgery, appendix and small bowel. Operative Surgery, Oxford University press,USA

10. Leventhal A, Pfau A (1978) Pharmacologic management of postoperative over-distention of the bladder. Surg Gynecol Obstet 146:347_348.

11. Cataldo PA, Senagore AJ (1991) Does alpha sympathetic blockade prevent urinary retention following anorectal surgery? Dis Colon Rectum 34:111_1116.

12. Lau H, Lam B (2004) Management of postoperative urinary retention: A randomized trial of in-out versus overnight catheterization. ANZ J Surg 74 658 661.

13. Scoma JA (1975) Catheterization in anorectal surgery. Arch Surg 110:1506.

14. Martinez OV, Civetta JM, Anderson K, Roger S, Murtha M et al. (1986) Bacteriuria in the catheterized surgical intensive care patient. Crit Care Med 14: 186-191.

15. Blandy J, Kaisary VA (2006) Prostate cancer. In: Urology. (6 $6^{\text {th }}$ edition) Wiley Blackwell publishers, USA. 